\title{
Adolescencia rural indígena, escuela y masculinidades"
}

\section{Indigenous rural adolescense, school and masculinities}

\author{
Lourdes Consuelo Pacheco-Ladrón de Guevara** \\ Universidad Autónoma de Nayarit, Tepic, México.
}

Recibido: 6 de febrero de 2018. Aprobado: 24 de marzo de 2018 . DOI: 10.25100/lamanzanadeladiscordia.v13i1.6715

Artículo de investigación

\begin{abstract}
Resumen: Tanto la niñez como la adolescencia se consideran construcciones socioculturales enmarcadas en contextos especificos. De ahi que lejos de establecer parámetros genéricos sobre la adolescencia, su estudio particular permite especificar las formas de ser adolescente en situaciones concretas. En el presente documento se aborda la construcción de las masculinidades rurales indígenas desde la comunidad y la escuela telesecundaria, para lo cual se realizaron grupos focales y entrevistas a estudiantes indígenas de telesecundaria de la Sierra Madre Occidental durante los años 2010 y 2011. Se discute la importancia de abordar la construcción de las masculinidades adolescentes en el marco de la irrupción de la escuela telesecundaria en la zona indigena en función de que la escuela telesecundaria se ha convertido en el espacio de la adolescencia indigena rural. Se concluye que la escuela tiene posibilidades de convertirse en un espacio de discusión de las propuestas comunitarias tradicionales si es capaz de replantear la funcionalidad de las masculinidades en los contextos especificos a partir de las condiciones del grupo cultural.
\end{abstract}

Palabras clave: Telesecundarias; adolescencia; etnia.

\begin{abstract}
Both childhood and adolescence are considered sociocultural constructions framed within specific contexts. Hence, far from establishing generic parameters about adolescence, studying it in particular settings allows us to specify the ways of being a teenager in concrete situations. In this document we approach the construction of rural indigenous masculinities in the community and in televised high schools; for this purpose, focus groups and interviews were conducted with indigenous televised high school students of the west Sierra Madre during the years 2010 and 2011. We discuss the importance of addressing the construction of adolescent masculinity in the context of the emergence of televised high school in indigenous areas since televised high school has become the space of rural indigenous adolescence in Mexico. We conclude that these schools have the potential to become spaces for the discussion of traditional community proposals if they are able to rethink the functionality of masculinities in certain contexts by taking the specifics of each cultural group as a point of departure.
\end{abstract}

Keywords: Televised high-school; adolescence; ethnicity.

\footnotetext{
*Este artículo presenta resultados de la investigación “Trayectorias juveniles rurales entre la escuela, el trabajo y la ciudadanía. Las posibilidades de agenciamiento de la juventud rural” que se llevó a cabo en la Universidad Autónoma de Nayarit, México, con apoyo del Consejo Nacional de Ciencia y Tecnología (CONACYT).

**Doctora en Ciencias Sociales. Investigadora de la Universidad Autónoma de Nayarit. Sus líneas de investigación son: Género y Pueblos indígenas. Miembro del Sistema Nacional de Investigadores (SNI), CONACYT-México. Correo electrónico: lpacheco_1@yahoo.com.
} 


\section{Introducción}

La adolescencia se considera la primera etapa de la juventud, sin embargo, en la actualidad, los estudios de juventud dan cuenta de la temporalidad de las consideraciones de la juventud ya que adquiere connotaciones de tiempo y espacio diferenciado por género y depende de los valores asociados a lo que se considera deseable para este grupo de edad (Feixa, 1998). La juventud como categoría se adhiere a la diversidad de los sujetos, a los ritos y límites que los marcan.

Tradicionalmente, la adolescencia se considera una etapa de la vida marcada por los cambios biopsicológicos que marcan la transición de la niñez a la juventud, propiamente dicha. Se asocia a cambios físicos, transformaciones del cuerpo, emocionales, sexuales y psicológicas.

[...] la adolescencia alude a una categoría biológico-psicológica, en la que resulta precisamente la "edad biológica", es decir, los cambios físicoemocionales, que caracterizan al adolescente como un sujeto inacabado. Su contraparte será el joven que es una categoría socio-cultural producto de procesos sociales, por lo que la juventud será una "edad social" (Nateras, 2002, p. 10)

Sin embargo, la adolescencia indígena rural está lejos de ser tan sólo una edad biológica, porque ella alude a un proceso social debido a que es la etapa en la cual los miembros de la comunidad establecen el lugar social de las personas. El desarrollo de las características individuales de cada adolescente es asumido de manera comunitaria en los espacios establecidos por la comunidad. A primera vista, las mujeres son homogeneizadas bajo el destino de la maternidad y las funciones asociadas a ello, sin embargo, al interior se encuentra un proceso de diferenciación que tiene que ver con las características (habilidades, carácter) de las mujeres y posteriormente, con la situación de conyugalidad. Los hombres son incorporados a las diversas funciones comunitarias desde las actividades capaces de desarrollar, el carácter y la posibilidad de asumir compromisos. Se puede decir que el destino genérico de los varones es el trabajo, entendido tanto como trabajo comunitario como ocupación por dinero, lo cual puede incluir una gran cantidad de modalidades de empleo.
El trabajo comunitario, a su vez, le permitirá arribar a la forma de ser adulto.

La adolescencia indígena adquiere características específicas debido a la situación propia de las comunidades indígenas, los procesos sociales en que se ven inmersos los adolescentes al interior de las comunidades y las interacciones con el mundo mestizo.

La adolescencia en las comunidades indígenas no se encuentra marcada por los cambios psicológicos, sino por la socialidad a que son constreñidos los y las adolescentes en aras de incrementar su participación en la solución de procesos colectivos de mantenimiento comunitario. De ahí que la adolescencia se configure como el espacio de introducción a la participación comunitaria, un proceso social que trasciende las dimensiones biológicas o psicológicas.

Lo anterior no significa la ausencia de cambios biológicos o psicológicos, sino que la carga comunitaria se sobrepone a lo primero. Esta idea también puede expresarse diciendo que la fuerza de la comunidad se impone a los procesos individuales de quienes las componen. Las comunidades establecen los órdenes a partir de los cuales los adolescentes hombres y mujeres deben transitar a la adultez. Desde este punto de vista es imposible hablar de una única forma de adolescencia indígena rural, ya que los procesos culturales de inmersión comunitaria son diversos a lo largo de las regiones indígenas del país, de ahí que se debe hacer referencia a las adolescencias, en plural.

Las adolescencias rurales indígenas están marcadas por la interacción de la comunidad en la que viven con el mundo mestizo. Ello significa que la mayor o menor proximidad con lugares urbanos marcará las dinámicas establecidas para los y las adolescentes al interior de la comunidad.

\section{Adolescencia y escuela}

Las adolescencias indígenas transcurrían tradicionalmente dentro de los cánones establecidos por la comunidad. Sin embargo, el establecimiento de las escuelas telesecundarias indígenas se convirtió en una irrupción dentro de las amplias zonas indígenas del México contemporáneo. La adolescencia indígena termina la educación formal en el umbral de los seis años de escolaridad, puesto que el siste- 
ma de educación bilingüe establecido en México, sólo cubría ese nivel.

Ante la imposibilidad de establecer escuelas secundarias para garantizar la educación básica a la población indígena, en amplios sectores del país se estableció la telesecundaria como una manera de ampliar la cobertura educativa a partir de un modelo basado en la utilización de la televisión en la función educadora. Sin embargo, a pesar de estar destinada mayoritariamente a zonas indígenas, la educación de telesecundarias no fue definida como educación bilingüe, ni perteneciente al campo de la educación destinada a pueblos indígenas. Ello implicó el establecimiento de programas generales elaborados para adolescentes mestizos, socializados generalmente, en regiones urbanas (Pacheco, 2006).

El establecimiento de las telesecundarias en el medio indígena proporcionó la posibilidad de contar con establecimientos institucionalizados por la civilización mestiza en el seno mismo de las zonas indígenas. La telesecundaria fue una continuación de la educación inicial y la educación primaria cuyos propósitos eran ampliar la cobertura a zonas marginales e incorporar a la adolescencia indígena a este nivel de la educación básica. La escuela junto con la clínica médica del sector salud son las instituciones visibles del modelo civilizatorio mestizo en las áreas indígenas (Levinson, 2002).

La investigación tuvo como objetivo analizar las posibilidades de las escuelas telesecundarias en el ámbito rural para abrir espacios en torno a la discusión de las masculinidades, para lo cual se realizó investigación de campo en comunidades indígenas Náyeri y Wixarika en el Estado de Nayarit.

\section{Masculinidad y feminidad entre los y las wixa- ritari}

El grupo cutural Wixaritari se ubica en la Sierra de Nayarit, Jalisco, Durango y Zacatecas donde tienen las poblaciones más importantes, caracterizadas por contar con gobiernos tradicionales: en Jalisco: Tateikie (San Andrés Cohamiata), Waut+a (San Sebastian), T+apurie (Santa Cantarina) y Tutsipa (Tuxpan de Bolaños). En Nayarit: Xatsitsarie (Guadalupe Ocotán). Se reconoce sin embargo, un territorio más amplio marcado por la geografía sagrada desde el desierto de Real de Catorce, donde se ubica
Wirikuta, (el lugar del nacimiento del sol) hasta el lago de Chapala en Jalisco.

En el panteón wixaritari existen tanto padres como madres con asignaciones diferentes. Ello implica que el principio masculino y femenino tiene expresión a través de distintos significados. El sistema sagrado se compone por un esquema de parentesco donde los elementos fundantes se convierten en parte de los ancestros. De esta manera, al ancestro fuego se le llama Tatewari: nuestro abuelo; el sol es Tayeu: nuestro padre sol y el venado en cuanto ancestro se llama Ta Matsi: nuestro hermano mayor. Al mismo tiempo se tienen ancestros femeninos como Nakawe: la abuela creadora del universo; $\mathrm{Ha}$ ramara: la madre del mar; Ta tei ut+anaka: la madre del maíz y otras más (Carrillo, 2008).

En la leyenda sobre Watakame (el coamilero) se cuenta que sembrar la tierra es una actividad donde intervienen tanto los ancestros como los seres humanos. Nakawe, la abuela creadora del universo, enseñó a Watakame a reconocer los cinco puntos cardinales de la cosmogonía sagrada wixaritari y también le enseñó a conservar las semillas y a sembrar. En el relato, Watakame salva a una perrita de la inundanción del mar, siguendo las indicaciones de la abuela Nakawe. Después de coamilear, Watakame encuentra tortillas dentro de su casa, por lo que decide espiar para descubrir lo que ocurre en su ausencia. Entonces encuentra que la perrita es en realidad una mujer.

El mito, fija los lugares de hombres y mujeres wixaritari dentro de la cultura, puesto que asigna el lugar del trabajo agrícola para los varones y el trabajo de la transformación del maíz en alimento a las mujeres. Al mismo tiempo, asigna al varón coamilero la capacidad de comunicarse con los ancestros.

\section{Las masculinidades en el ámbito rural indígena}

Las ruralidades conservan y reproducen el modelo tradicional de masculinidad y feminidad, derivado de la introducción de patrones en la época de la colonia y resignificados por los pueblos indígenas (Pérez, 2011) a partir de sus propias prácticas y simbologías contenidas en su tradición a partir de la multiplicidad de trabajos que deben realizarse en el ámbito rural. Generalmente a los varones se les socializa en actividades donde se utiliza mayormente 
la fuerza, en tanto que las mujeres son socializadas para la vida familiar y reproductiva. En el ámbito rural, ambas actividades son necesarias para la sobrevivencia del grupo.

Si bien las masculinidades en el medio indígena deben hacer hincapié en las diversas influencias que los diversos estadios de la occidentalización han causado en los pueblos indios (Díaz-Cervantes, 2014), se reconoce la existencia de una matriz simbólica a partir de los cuales se resignifican en las actuales prácticas organizaciones comunitarias.

La práctica de la masculinidad tradicional está vinculada a las destrezas en el manejo de animales, el uso de armas como el machete y la demostración de valentía vinculada a la simbología del grupo, por ejemplo, cazar venado, pasar la noche en una cueva, etc. De ahí que a los varones se les asignen diversas funciones, mientras que las mujeres participan de la comunidad a partir de su asignación a las actividades vinculadas a la reproducción.

Entre los procesos sociales en que se ven inmersos los adolescentes al interior de las comunidades se tiene:

- La asignación de tareas comunitarias a partir del crecimiento y el sexo. En las comunidades, los adolescentes son destinados a diversas actividades dependiendo de las posibilidades de realizarlas. Esto significa que al niño varón le son encomendadas actividades agrícolas dependiendo de las fuerzas que tiene para realizarlas. Por ejemplo, al momento en que las fuerzas físicas le permiten controlar un machete, puede convertirse en cortador de caña. También puede ser destinado a cuidar animales (vacas o chivos), a pescar en ríos y arroyos sin la supervisión de un adulto.

- Lo mismo ocurre con las mujeres. Si desde la pequeña edad pueden convertirse en pequeñas madres cuidadoras de sus hermanos pequeños, en la adolescencia las chicas empiezan a incursionar en tareas de mayor complejidad preparatorias de las actividades futuras que realizarán en tanto reproductoras de la vida. Por ejemplo, llevar a cabo partes del proceso de elaboración de tortillas, lo cual implica una serie de actividades complejas tales como elaborar nixtamal, poner leña, moler maíz y tortear.
- En cuanto los cargos comunitarios, los adolescentes indígenas inician el proceso de introducción a la vida de responsabilidades comunitarias desde un lugar específico. En las comunidades Nayerij de Nayarit, los adolescentes varones forman parte de los grupos de borrados con que el grupo cultural celebra la Judea cora de la Sierra del Nayar, principal festividad de este grupo cultural y síntesis del sistema cultural vinculado al ciclo agrícola. Si bien en el proceso participan niños, su ingreso se entiende dentro de un proceso lúdico de entrenamiento. En cambio, para los adolescentes varones es parte de la obligatoriedad del cumplimiento de la costumbre.

- En las comunidades wixarikas, los adolescentes varones inician participando como los guardianes del cuidado del maíz en ceremonias donde el ciclo agrícola estructura la identidad comunitaria. En ambas culturas, los adolescentes varones son considerados parte fundamental de tales ceremonias, son las formas como las comunidades marcan los ciclos transicionales entre la niñez y la madurez indígena.

- Las adolescentes mujeres participan en la comunidad desde los rasgos asignados a partir del género. Esto implica responsabilizarse de actividades reproductivas dentro de la familia de origen como un entrenamiento para la construcción de su propia familia de destino. Para las mujeres, son los rasgos biológicos los que marcan el límite de la niñez y la entrada a la madurez ya que la aparición de la menarquia posiciona a la niñaadolescente dentro del campo de las mujeres aptas para concebir. Este es el signo para formar una nueva familia $y$, por lo tanto, asumir nuevos roles.

- En las comunidades Nayerij las niñas son consideradas vírgenes, por lo que a partir de esta consideración participan en rituales de la costumbre: son las malinches dentro de Las Pachitas, ceremonia ritual con que inicia la preparación de la Semana Santa, cuarenta días antes del viernes santo.

- En las comunidades Wixarikas, las adolescentes son seleccionadas por los cantadores para participar en la ceremonia del Esquite, con que la comunidad pide permiso a la Madre Olíanaka 
para sembrar el maíz y se invoca a las Madres de la lluvia para tener una buena cosecha. Las adolescentes son quienes tuestan el maíz ceremonial que será ofrecido en el altar de las madres.

\section{La familia de la adolescencia indígena}

La familia de la adolescencia indígena rural está caracterizada por dos elementos: Por una parte, se trata de familias en ámbitos rurales cuya base de sostenimiento es la agricultura de temporal, alrededor de ella se desarrollarán otras actividades que complementarán los bienes alimenticios y de sostenimiento general. La segunda característica es la condición étnica que hace referencia a participar de un conjunto de características culturales propias diferentes de las compartidas por la sociedad mestiza.

En las comunidades indígenas rurales, la participación productiva de la familia es integral, se trata de un trabajo realizado por todos los miembros de la familia de acuerdo a las condiciones de sexo y edad. Así, los niños van incorporándose a actividades relacionadas con la siembra en tareas de ayuda a los mayores (acarreo de semilla, arranque de hierba, etc.) en tanto que las niñas se incorporan a diversas labores vinculadas al trabajo realizado por las mujeres tanto para la transformación de los alimentos agrícolas como para la reproducción de animales doméstico o conservación de plantas (acarreo de agua, alimentar animales, etc.)

En síntesis, la familia rural indígena se caracteriza por los lazos de cooperación y reciprocidad en que ocurre la socialización infantil y juvenil tanto entre los miembros del grupo como con el entorno. Si bien anteriormente la familia tradicional indígena se caracterizaba por el desarrollo preponderante de actividades agrícolas para el autoconsumo, actualmente los diversos miembros participan en actividades no necesariamente vinculadas a la agricultura, como la producción de artesanías o el comercio. Cada vez más realizan una diversidad de actividades vinculadas al mercado.

El trabajo y la prestación de servicios que realizan los diversos miembros de la comunidad tienen que ver con el autosostenimiento del grupo, ya sea porque aporte trabajo individual a tareas comunes o porque se aporte trabajo individual para la propia reproducción familiar. Tanto las mujeres, como niños y ancianos realizan diversas tareas relacionadas con su edad, su condición y fortalezas que tienden hacia el mantenimiento del grupo y la reproducción colectiva. La faena, por ejemplo, de acarrear agua de fuentes lejanas o de recopilarla de manantiales o llaves externas a la vivienda, realizadas por los miembros del grupo se convierte en una actividad central para los propósitos de la reproducción.

Lo mismo puede decirse de las actividades de recolección de frutos silvestres como de cultivos domésticos, los cuales son parte de la dieta diaria de los pueblos indígenas. Las mujeres complementan los alimentos obtenidos en el mercado con el cultivo de especies domésticas en traspatios, la cría de animales de corral o captura de animales pequeños.

Los pueblos indígenas poseen un sistema de socialización en el trabajo caracterizado por estar dirigido a la construcción de comunidad. Tanto el trabajo realizado por hombres, como por mujeres tiene ese fin en los distintos periodos de edad. El trabajo comunitario no sólo produce bienes sino que se instala en la construcción de sentido comunitario: otorga sentido de pertenencia al grupo; contribuye a la construcción simbólica del colectivo puesto que tanto la agricultura como los textiles son parte de las actividades enseñadas por los dioses de la tradición a hombres y mujeres; la incorporación al trabajo son señales de la madurez biológica y social de los indígenas; la apropiación de los productos del trabajo es colectiva; el trabajo es parte de la pedagogía comunitaria puesto que la interrelación generacional permite la transmisión de saberes entre miembros de distintas generaciones.

\section{Los trabajos asignados a los adolescentes indí- genas}

Durante el año de 2010 y 2011 se realizó la investigación de campo en comunidades indígenas dentro de las comunidades Nayerij o coras de Nayarit, específicamente en dos localidades: Santa Cruz de Gaybel y El Maguey, ambas del municipio de El Nayar, comunidades de la Sierra Madre Occidental. La comunidad Cora de Santa Cruz de Guaybel pertenece a la Región zona Cora baja. Cuenta con 397 habitantes, de los cuales 213 son hombres y 184 son mujeres (Ayuntamiento de El Nayar, 2008), se en- 
cuentra situada en la orilla de la carretera Estación Ruiz - Zacatecas, en el tramo San Pedro Ixcatan Mesa del Nayar. La comunidad de El Maguey se encuentra en ese mismo camino, a dos kilómetros de distancia.

La información fue recuperada dentro del contexto de las y los adolescentes con la finalidad de interpretarlos en el espacio y tiempo en que se desarrollan cotidianamente los adolescentes ya que se parte del principio de que el contexto es parte de la información. El estudio etnográfico se utilizó a fin de comprender las creencias, valores, perspectivas y motivaciones (Bisquerra, 2004) de las y los adolescentes. Para el análisis de la información se utilizó el método de codificación abierta, encaminada a encontrar el significado de los datos (Abréu, García-Nieto y Pérez, 2007)

Los adolescentes hombres y mujeres identificaron los siguientes trabajos realizados por ellos mismos, véase la Tabla 1:

Tabla 1: Actividades identificadas por adolescentes mujeres y varones

\begin{tabular}{|c|c|c|}
\hline Ámbito & Adolescentes varones & Adolescentes mujeres \\
\hline Casa & Acarrean leña, agua, hacen mandados & $\begin{array}{l}\text { Tortean, hacen aseo, lavan ropa, lavan trastes, ba- } \\
\text { rren, riegan plantas, cuidan animales, dan de comer } \\
\text { al burro, van a la leña, al agua, muelen maíz, peinan, } \\
\text { cambian y bañan a sus hermanos pequeños, recogen } \\
\text { la basura, despiojan, siembran y cuidan flores }\end{array}$ \\
\hline Agricultura & $\begin{array}{l}\text { Van al campo con hombres adultos de su } \\
\text { familia, coamilean, plantan árboles, tum- } \\
\text { ban coamil, machetean, cuidan vacas, re- } \\
\text { fuerzan cercos, piscan, rosean el campo. } \\
\text { Ponen trampas para cazar animales }\end{array}$ & $\begin{array}{l}\text { Ayudan en la época de siembra y a recoger hojas } \\
\text { cuando es la cosecha. Recolectan nopales, nanchis. } \\
\text { Cortan frutas de los árboles (mangos, guayabas, etc.) }\end{array}$ \\
\hline Ocupaciones & $\begin{array}{l}\text { Ayudan a hacer mesas y sillas, huaraches, } \\
\text { ladrillos, sombreros, cintos }\end{array}$ & $\begin{array}{l}\text { Ayudan a su mamá/papá a vender fruta, comida, ha- } \\
\text { cen artesanía, }\end{array}$ \\
\hline Escuela & $\begin{array}{l}\text { Van a la escuela, juegan en la cancha, ha- } \\
\text { cen tareas }\end{array}$ & Van a la escuela, juegan en la cancha, hacen tareas \\
\hline Juegos & $\begin{array}{l}\text { Juegan en el campo, suben a los árboles, } \\
\text { canicas }\end{array}$ & $\begin{array}{l}\text { Juegan voleybol, se peinan, cantan, juegan en el } \\
\text { campo }\end{array}$ \\
\hline
\end{tabular}

Fuente: Elaboración propia.

Identificaron como prototipos de hombres y mujeres adultas los siguientes:

- Cómo debe ser un hombre: Fuerte, trabajador, que cuide bien a su esposa, responsable, guapo, debe de tomar, ser ranchero, cuidar las vacas, grande, alto, serio, corajudo.
- Cómo debe ser una mujer: Bonita, trabajadora, limpia, honesta, platicadora, amigable, chismosa, débil, lista, amorosa.

\section{Masculinidades como continuidades}

Si bien, algunos y algunas adolescentes indígenas generan expectativas de su vida futura a partir 
de la educación, la tradición y la comunidad adquieren un peso específico a la hora de plantear el futuro, ya que las actividades y ocupaciones de la tradición se convierten en lo posible, prácticamente en lo que seguramente realizarán, más allá de los deseos de las y los adolescentes. De ahí que algunas adolescentes establecen como plan de futuro ser amas de casa, en tanto que los varones expresan como plan de vida trabajar en el campo.

Algunas adolescentes mujeres plantean ser maestras, enfermeras o doctoras. Los hombres, en cambio, proponer ser soldados o doctor.

Las expectativas de las y los adolescentes están vinculadas a las actividades que se realizan en la comunidad y a las ocupaciones de quienes llegan a la comunidad con otros empleos, tal es el caso de quienes expresan ser soldados, ya que, debido a la mayor presencia de miembros del Ejército en las comunidades, los jóvenes ven como posibilidad real enrolarse en el Ejército. Ello les garantiza un empleo, salario y seguridad social.

Las partidas militares se han establecido en las cercanías de las comunidades indígenas, sobre todo en aquellas comunidades que cuentan con pista de aterrizaje, ya que el Ejército custodia el uso de las pistas. Ello ha ocasionado una mayor visibilidad del Ejército para las juventudes y adolescencias indígenas, que ven el acceso al Ejército como algo posible. Las mujeres, en cambio, tienen como ejemplo a las maestras y enfermeras que llegan a las clínicas o son parte de las brigadas de salud que se despliega en las comunidades indígenas.

\section{Reflexiones finales: masculinidades y escuela}

De acuerdo con el estudio realizado, la irrupción de la telesecundaria en las zonas indígenas de estudio abre un espacio nuevo para el transcurrir de la adolescencia indígena debido a que acerca los y las jóvenes a nuevas formas de pensar el futuro. Sin embargo, las condiciones del medio aunadas a la falta de especificidad de la escuela telesecundaria para trabajar en el medio indígena minimizan la acción de esta escuela.

La telesecundaria en las zonas indígenas de las comunidades estudiadas tiene pocas posibilidades de crear nuevos contextos a partir de los cuales las/ os adolescentes indígenas planteen nuevas formas de lazos sociales, relaciones entre hombres y mujeres y agrupamientos que les permita plantearse expectativas diferentes a las comunitarias y a las que propagan los medios de comunicación. Por el contrario, las diversas necesidades de las comunidades rurales acentúan las prácticas de las masculinidades como forma de sobrevivencia. A ello debe agregarse la introducción de los medios de comunicación, que acentúan las masculinidades tradicionales vinculadas al poder, la virilidad, la gallardía, lo cual en las comunidades indígenas se convierte en permiso para andar donde quieran, poder hablar con voz fuerte, tomar cerveza y golpear a las mujeres y niños, entre otros.

La simbología contenida en la cosmogonía del grupo Wixaritari contribuiría a construir relaciones más igualitarias, pues lejos de ser una cultura de predominancia masculina, los principios femeninos se encuentran expresados en ancestros femeninos de igual valor que los ancestros masculinos. De ahí que los y las jóvenes cuentan con posibilidades simbólicas de encontrar nuevos significados a sus relaciones.

No es posible generalizar la información derivada del estudio realizado, sin embargo, es preciso reflexionar acerca de las posibilidades de la telesecundaria en zonas indígenas a fin de replantear la funcionalidad de las masculinidades y feminidades tanto simbólicas como reales, en ese sentido, la escuela telesecundaria en determinados contextos tendría posibilidades de convertirse en un lugar definitorio para la adolescencia tanto masculina como femenina. Ello sería posible para dejar de ser sólo un lugar transicional del mundo indígena comunitario al mundo mestizo como aspiración posible.

\section{Referencias bibliográficas}

Ayuntamiento de El Nayar. (2008). Plan Municipal de Desarrollo del municipio de El Nayar. Jesús María: Ayuntamiento de El Nayar.

Abréu, Jaime; García-Nieto, Antonio y Pérez, Ana. (2007). Evolución de la teoría fundamentada como técnica de análisis cualitativo. Madrid: Centro de Investigaciones Sociológicas (CIS).

Bisquerra, Rafael. (2004). Metodología de la investigación Educativa. Madrid: Editorial La Muralla, S.A.

Carrillo, Tutupika. (2008). Feminidad y masculinidad en la mitología Wixarika (Tesis de especialización). 
Universidad Autónoma de Nayarit, Tepic, México. Díaz-Cervantes, Rufino. (2014). La perspectiva de género en la comprensión de la masculinidad y la sobrevivencia indígena en México. Agricultura, sociedad y desarrollo, 11(3), 359-378. Recuperado de http://www.scielo.org.mx/scielo.php?script=sci arttext\&pid $=$ S1870-54722014000300006\&lng $=$ es\& nrm=iso.

Feixa, Carlos. (1998). El reloj de arena. Culturas juveniles en México. México: Instituto Mexicano de la Juventud.

Levinson, Bradley. (2002). Todos somos iguales: cultura y aspiración estudiantil en una escuela secundaria mexicana (Susana Moreno y María Arruti, Trads.).
México: Aula XXI y Santillana. (Obra original publicada en 2001).

Nateras, Alfredo (Coord.). (2002). Jóvenes, culturas e identidades urbanas. México: Universidad Autónoma Metropolitana.

Pacheco, Lourdes. (2006). Los retos de la educación en los adolescentes rurales en México. En Lesvia Rosas (Coord.), La educación rural en México en el siglo XXI (pp. 279-306). México: Centro de Estudios Educativos.

Pérez, Elia. (2011). Dificultades y contradicciones en la configuración de las identidades nahuas de tres generaciones de hombres de la Sierra Norte de Puebla: Estudio de Caso (Tesis doctoral). Universidad Complutense, Madrid, España. Recuperado de https:// eprints.ucm.es/12025/1/T32599.pdf. 